\title{
Haemangioendothelioma of Oral Cavity: A Rare Entity with Unusual Location
}

\author{
Patel Nikita Rajendrakumar1, Jagtap Kiran Suresh1, Anjali Wadhwa2, Pravin Mukhi2 \\ and Pranjal Anil Patel 3
}

\begin{abstract}
Hemangioendothelioma is a rare, locally aggressive vascular tumor, originating from soft tissue, bone, skin, and organs such as liver or lung and exceptionally the oral cavity. It is usually benign but can show variable grades of malignancy. Most of the cases of oral epithelioid hemangioendotheliomas are asymptomatic, and diagnosis is hampered by the fact that the histological features somewhat overlap with hemangioma and angiosarcoma, with epithelioid cells, intracytoplasmic vacuoles, low mitotic activity, and rarely necrosis. Immunohistochemical analysis is required to rule out carcinoma or other epithelioid vascular neoplasms. Here, we report a very rare case of oral haemangioendothelioma in a 41-year female patient which presented as an exophytic, painless, mass on the labial gingiva, clinically diagnosed as pyogenic granuloma, which presented a diagnostically challenging histological picture, which required immunohistochemistry to achieve at the correct diagnosis.
\end{abstract}

Key Words: Vascular tumors, Oral haemangioendothelioma, Immunohistochemistry.

\section{INTRODUCTION}

Hemangioendothelioma is a vascular tumor of intermediate grade between hemangioma and angiosarcoma, characterised by proliferating neoplastic endothelial cells. ${ }^{1}$ The term hemangioendothelioma was introduced by Borrmann, who first proposed the concept of vascular neoplasms with intermediate or low malignant potential. ${ }^{1}$ Three histological types of hemangioendothelioma are known: kaposiform, hobnail (or Dabskaretiform), and epithelioid. ${ }^{2}$

The epithelioid hemangioendothelioma (EHE) type was first described by Weiss and Enzinger in 1982 as an angiocentric neoplasm characterised by neoplastic proliferation of epithelioid or histiocytoid endothelial cells, showing eosinophilic vacuolated cytoplasm, and occasionally, fusiform cells. ${ }^{2}$ The tumor is usually located in the soft tissue of the extremities and less frequently in the liver, lung, pleura, bones, skin, lymph nodes, and central nervous system. ${ }^{2}$ EHE is rarely diagnosed in the head and neck region, and even more rarely in the oral cavity. ${ }^{3}$

1 Department of Oral Pathology and Microbiology / Oral and Maxillofacial Surgery2, SMBT Institute of Dental Sciences and Research, Nashik, Maharashtra 422403, India

3 Department of General Medicine, Suyash Multispeciality Hospital, Nashik, Maharashtra 422403, India

Correspondence: Dr. Nikita Rajendrakumar Patel, Department of Oral Pathology and Microbiology, SMBT Institute of Dental Sciences and Research, Nashik, Maharashtra 422403, India

E-mail: patelnikitar.15@gmail.com

Received: June 11, 2018; Revised: September 11, 2018; Accepted: November 14, 2018
We report a very rare case of EHE occurring in the oral cavity in a 41-year female patient.

\section{CASE REPORT}

A 41-year female patient visited the Department of Oral Medicine and Oral Radiology with the chief complaint of painless, red, ulcerated, friable, and progressively enlarging mass for 1 year. On intraoral examination, the lesion was seen on labial gingiva of maxillary arch from right second premolar to left lateral incisor extending over the hard palate and measuring approximately $3 \times 4 \mathrm{~cm}$ in dimension. Similar small lesions were seen on mandibular labial gingiva measuring approximately $1 \times 2 \mathrm{~cm}$ in dimension extending from right central incisor to left lateral incisor (Figures 1 and 2). On palpation, there was associated bleeding.

No any other relevant clinical findings were noted. Patient was advised investigations such as complete haemogram, orthopantomograph (OPG) (Figure 3) and excisional biopsy. A complete blood count was within normal range. Radiographic examination showed bone resorption in the upper and lower anterior region.

Based on clinical findings, provisional diagnosis of pyogenic granuloma was made. Patient consent was taken and excisional biopsy was done. Microscopical findings revealed proliferation of endothelial cells in the form of sheets and cords. Nuclear atypia was noted. Areas of necrosis, proliferation of spindle-shaped endothelial cells, bizzare shaped vascular spaces with thrombi or phlebolith were also detected. Based on histopathological findings, diagnosis of intermediate grade of vascular tumor was made. For confirmatory diagnosis, immunohistochemistry was done, which showed 


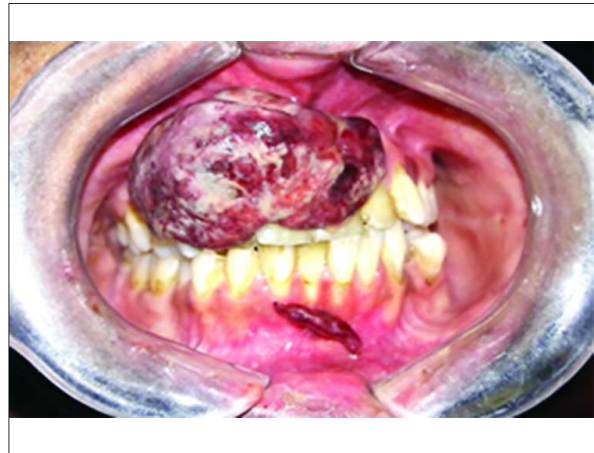

Figure 1: Lesion seen over labial gingiva of maxillary and mandibular arch palate.

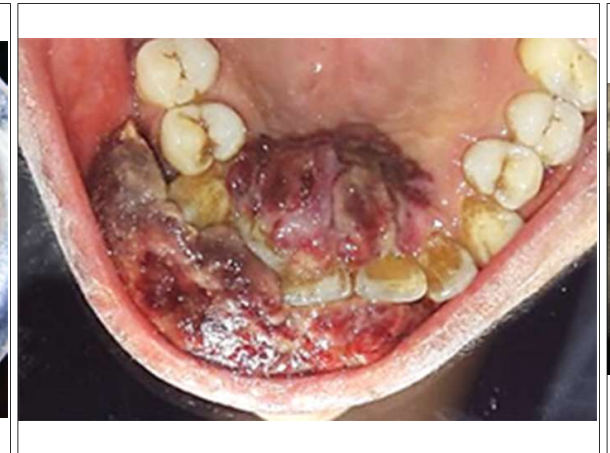

Figure 2: Lesion extending upto palate.

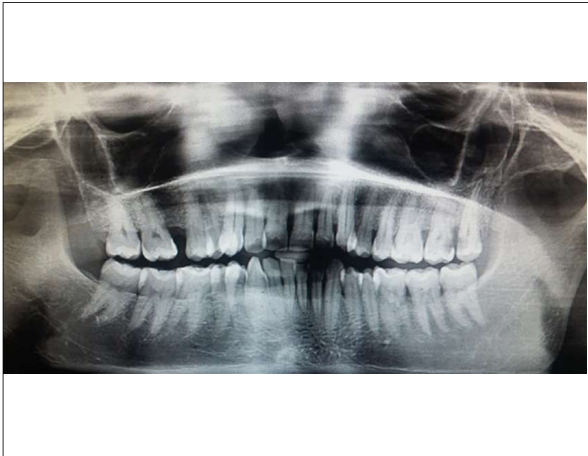

Figure 3: OPG presented bone resorption in anterior region of upper and lower arch.

positivity for CD 31 marker and vimentin (Figure 4a-d). To rule out malignancy, cytokeratin, P63 and P40 were done which showed negativity; thus possibility of carcinoma was ruled out. Markers such as smooth muscle actin (SMA) and S-100 were done to rule out smooth muscle and neuronal tumors. Based on immunohistochemical findings, final diagnosis of haemangioendothelioma was made.

\section{DISCUSSION}

The term hemangioendothelioma was originally coined by Mallory in 1908, to include all proliferations that he considered as originating from endothelial cells of blood vessels. ${ }^{4}$

Hemangioendothelioma is characterised by endothelial cell proliferation around a vascular lumen. It has a biological behaviour that falls somewhere between the benign haemangioma and malignant angiosarcoma. Enzinger and Weiss have categorised hemangio-endothelioma into epithelioid, Kaposiform, hobnail, composite and epithelioid sarcoma-like hemangio-endothelioma. 5 Hamangioendothelioma is usually seen during second and third decade of life and females are affected more than males. ${ }^{6}$ It generally occurs in soft tissue and internal organs, with head and neck being an uncommon site. ${ }^{7}$ Primary lesions of oral cavity though not common, have been reported in a variety of locations, including lips, palate, gingiva, tongue and centrally within maxilla and mandible. Localised swellings may be the primary manifestation of the lesion. 6 In the present case the patient reported was 41-year female. Localised swelling was seen on rare location, that is gingiva and extending on to the palate and a small lesion on mandibular gingiva. According to literature, radiographic features, in about $25 \%$ of cases, show resorption of underlying bone. ${ }^{2}$ Radiological investigations are usually nonspecific, showing a poorly defined osteolytic lesion, with resorption or destruction of the underlying bone. ${ }^{2}$ In the current case also redetected bone resorption. Many cases of hemangio-endothelioma of oral cavity have been clinically diagnosed as benign lesions such as pyogenic

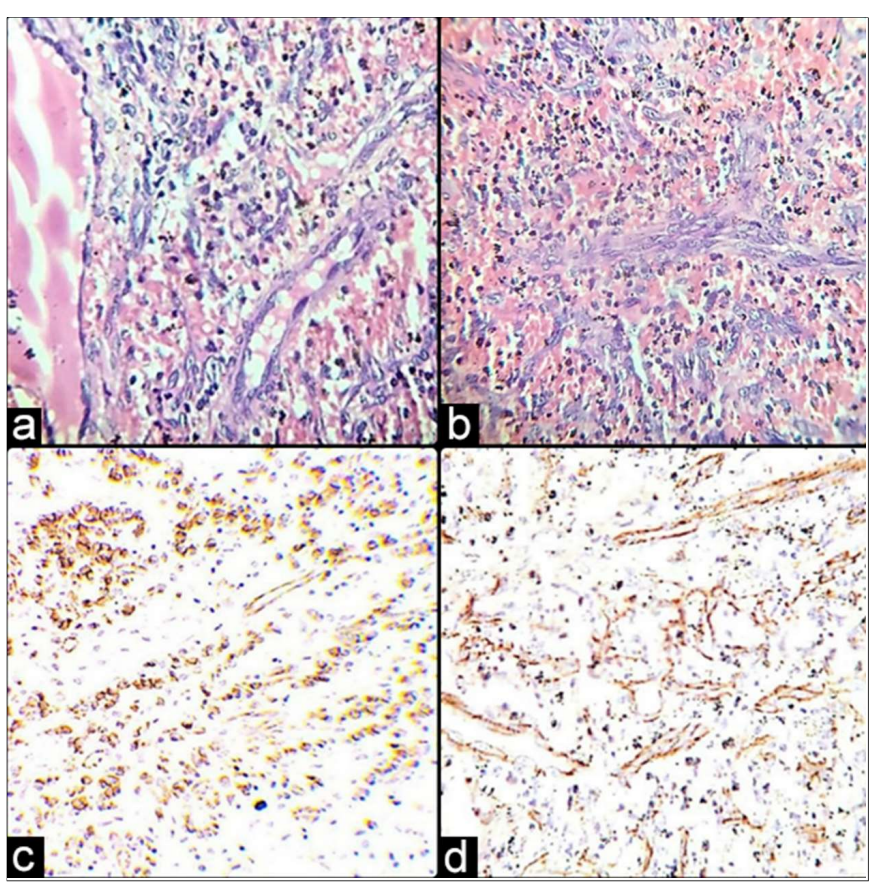

Figure 4: (a) Proliferation of dilated vessels with thrombi and phleboliths. (b) Proliferation of endothelial cells in sheets and cords. (c) CD 31 positivity of endothelial cell proliferation. (d) Proliferation of vascular channels.

granuloma, fibroma, peripheral giant cell granuloma, peripheral ossifying fibroma, inflammatory fibrous hyperplasia and necrotising ulcerative gingivitis. ${ }^{2}$ In this case, differential diagnosis included pyogenic granuloma, fibroma and peripheral giant cell granuloma. The histological features of haemangioendothelioma show proliferation of capillary size vessels with proliferation of epithelioid-like endothelial cells. These vessels are intermixed with solid sheets of epithelioid cells or spindle-shaped cells. Significant cellular atypia, areas of necrosis, high proportion of spindle cell proliferation, presence of mitotic figures indicate more aggressiveness of the tumor. ${ }^{3}$ Primitive-appearing vascular channels are formed with erythrocytes occasionally seen in the lumina. ${ }^{8}$ Similar, microscopic findings were noted in this case. No mitotic figures were seen which suggested intermediate nature of the tumor. To give the 
confirmatory diagnosis, immunohistochemistry was done which showed positivity for CD-31 and vimentin. This led to confirmation of vascular tumor. cytokeratin, and P63, were done to rule out the possibility of carcinoma; and SMA and S-100 were done in order to rule out smooth muscle and neuronal origin. The majority of intraoral EHE lesions are immunoreactive for CD34, CD31, factor VIII-related antyen and vimentin, which characterise the endothelial origin of this entity. ${ }^{9}$ Similarly, in the present case, the endothelial cells were positive for CD 31. As the morphological characteristics of EHE are nonspecific, the lesion can be confused with many others, including hemangioma, squamous cell carcinoma, or melanoma. Immunohistochemical tests are important for establishing the correct diagnosis, with the majority of intraoral EHE lesions being immunoreactive to CD34, CD31, factor VIII-related antigen, and vimentin, highlighting the endothelial origin of the entity. ${ }^{3}$

In conclusion, haemangioendothelioma is extremely rare in oral cavity and is often misdiagnosed as benign reactive lesion by oral surgeons and thus misunderstanding the lesion may further impede the treatment outcome. Hence, the pathologists and the oral surgeons should be aware of the occurrence of such rare lesions in oral cavity. As the histological picture is controversial posing further diagnostic challenges, immunohistochemical analysis is mandatory for confirming the diagnosis in cases of oral haemangi-oendothelioma.

\section{REFERENCES}

1. Heera R, Cherian LM, Lav R, Ravikumar V. Hemangioendothelioma of palate: A case report with review of literature. J Oral Maxillofac Pathol 2017; 21:415-20.

2. Gordón-Núñez MA, Silva eM, Lopes MF, de Oliveira-Neto SF, Maia AP, Galvão HC. Intraoral epithelioid hemangioendothelioma: A case report and review of the literature. Med Oral Pathol Oral Cir Buccal 2010; 15:340-6.

3. Lacob A, Comisel S. Oral Epithelioid Hemangioendothelioma Unusual location of a rare entity. J Interdisciplin Med 2017; 2:349-53.

4. Requena L, Kutzner H. Hemangioendothelioma. Semin Diagn Pathol 2013; 30:29-44

5. Goldblum JR, Folpe AL, Weiss SW. Hemangioendothelioma: Vascular tumors of intermediate malignancy. In: De Francesco K, editor. Enzinger and Weiss's Soft Tissue Tumors. ed $6^{\text {th }}$. Philadelphia: Elsevier Saunders; 2014. 681-702.

6. Sivapathasundharam B, Gurvraj N, Rangnanathan K. Viral infections. In: Sivapathasundharam B. Ed. Shafer's textbook of oral pathology. ed. 8th. Elsevier 2016: p.219-20.

7. Gupta SC, Tewarson SL, Malhotra M. Hemangioendothelioma of paranasal sinuses with intracranial extension. Indian $J$ Otolaryngol Head Neck Surg 2006; 58:196-8.

8. Mohtasham N, Kharrazi AH, Jamshidi S, Jafarzadeh $H$. Epithelioid hemangioendothelioma of the oral cavity: A case report. J Oral Sci 2008; 50:219-23.

9. Ali S, Odell EW, Whaites E, Robinson PD, Challacombea SJ. Epithelioid haemangioendothelioma of the mandibular gingiva: Case report and literature review. Int J Surg Case Rep 2015; 14:194-8. 